\title{
Recommendations for Diagnosis, Treatment and Prevention of Pneumonia Associated with Mechanical Ventilation
}

\author{
Dra Mirta D Ambra* \\ Chairman of the World Academy Medical Sciences, Ministry of Health University of Buenos Aires Argentina, Argentina
} Received: 阱 July 14, 2018; Published: 阱July 25, 2018

*Corresponding author: Dra Mirta D Ambra, Ministry of Health University of Buenos Aires Argentina, C Argerich Hospital, Academy of Medicine and Science, Argentina

\begin{abstract}
A system and method for the non-invasive portable ultrasound-based measurement system of the Cardiovascular blood pressure is presented, wherein the blood pressure measurement model is calibrated to the simultaneous direct measurements by pressure transducers during the catheterization. The model is based on the image time series processing estimating the changes of the oscillating traceable regions via the introduction of the new ultrasound generalized M-Mode and the notion of the derived image. Once calibrated the system is ready to recalculate the pressure in the calibrated region, such as Left Atrium according to the subsequent non-invasive ultrasound measurements. The system has been successfully tested on animals (sheep) under subsequent catheterisations.
\end{abstract}

Keywords: Methodology; Diagnostic; Antibiotic; Antimicrobials; Polyvalent; Morbimortality; Leukocytosis; Gasometry; Pneumonia; Acinetobacter Baummanii

Abbreviations: CHF: Congestive Heart Failure; LAP: Left Atrium Pressure; RAP: Right Atrium Pressure; LVEDP: Left Ventricular End Diastolic Pressure; PAP: Pulmonary Artery Pressure; PCWP: Pulmonary Capillary Wedge Pressure; AFR: Atrial Flow Regulator; ICP: Intra-Cardiac (blood) Pressure; US: Ultrasound.

\section{Methods}

Data from the Infection Committee associated with the Health Care and Patient Safety (IACS-SP) of the Argentine Infectious Disease Society (SADI) and the Critical Infectiousness Committee (CIC) of the Argentine Intensive Therapy Society (SATI) were observed on the subject. A systematic bibliographic search was carried out, whose sources were Medline, Pubmed, Embase, LILACS, Ovid, and Cochrane. MESH terms were used in Spanish and English in the period 2002-2017, in the adult population. The types of studies were guidelines, systematic reviews, randomized clinical trials and observational studies. The most recognized current international guidelines (USA, United Kingdom, Canada, Latin America and Europe) were established as main bibliography, being adapted to the characteristics and epidemiology of our critical units.

\section{Initial Attention}

The clinical evaluation includes all patients under mechanical ventilation for more than 48 hours that begin with fever, leukocytosis, increased secretions and deterioration of gasometry. The clinical assessment scale of pulmonary infection CPIS is a score that can contribute to diagnosis and evolution of pneumonia. The British guidelines recommend also considering the increase in the requirement of oxygen objectified through PEEP (positive end expiratory pressure) or $\mathrm{PaO} 2$ / FIO2 (Blood pressure and inspired oxygen fraction). This data is considered key to the new epidemiological definition of the US Centers for Disease Control and Prevention. (CDC) and surveillance of the NAVM in a unit11. Complementary studies such as chest x-ray and, possibly, computerized axial tomography (CT) are essential to determine the presence of new pulmonary infiltrate, although they are often difficult to interpret. Early VAP is considered to be that manifested within four days of ventilation and late VAPM from the fifth day. This distinction is made with the objective of considering the microorganisms of the community in the early and the intra hospitalary in the late, as causal agents of the infection. However, in patients who have had previous hospitalization, use of antibiotics (ATB) or risk factors for infection associated with health care (IACS), the probability of carrying resistant microorganisms (MOR) is prioritized. causes of early NAVM in the first days of mechanical ventilatory assistance (MVA) [3]. There is no gold standard for the diagnosis of VAP. Clinical suspicion should determine the immediate sample taking, 3, 4 and the start of early antibiotic treatment, to then reassess the diagnosis and treatment at $72 \mathrm{~h} 4$, 5 , 7. With respect to the microbiological study, recent guidelines prioritize quantitative or semiquantitative samples over qualitative 
ones and there are discrepancies in the revenue between the distal samples (broncho-alveolar lavage protected brush) and proximal samples, such as the tracheal aspirate. In our opinion, any type of sample (distal or proximal, quantitative or semi-quantitative) can be analyzed, prioritizing the one that is available at the time of suspicion of pneumonia. A significant count is considered to be 103 $\mathrm{CFU} / \mathrm{ml}$, with a protected brush, $104 \mathrm{CFU} / \mathrm{ml}$ in broncho-alveolar lavage Inadequate empirical therapy has an impact on mortality.

The early and adequate administration of antibiotics is indispensable, since this measure decreases mortality by approximately $22 \%$, as has been demonstrated by Kumar. The empirical antibiotic scheme should be according to the local epidemiology, stratification by hospitalization days in critical units and hospital (early $\leq 4$ days or late $\geq 5$ days) and risk factors for MOR: use of antibiotics in the previous 90 days in rooms of high prevalence of resistance, more than 4 days of hospitalization or colonization by resistant germs such as carbapenemase producing enterobacteria, Acinetobacter Baummanii or Pseudomonas spp. In most of the critical units it is probable that, according to the local epidemiology, antibiotic treatment for late pneumonia with at least two drugs should be carried out. According to the 2016 VIHDA2 report, the main MOR producers of NAVM in 149 ICUs in the country were Pseudomonas Aeruginosa (23.3\%), Staphylococcus Aureus (17.75\%), Klebsiella Pneumoniae (15.9\%) and Acinetobacter Baummani (14.1\%). For the initial empiric treatment of early VAP, without risk factors for MOR, it is suggested to start treatment for community respiratory agents such as pneumococcus, Haemophilus influenzae, methicillin-sensitive Staphylococcus (SAMS) and Branhamella Catarrhalis using ampicillin-sulbactam (AMS), and that in our environment the pneumococcus continues to be sensitive to penicillin. For late VAM with risk factors for MOR, the antibiotics to be considered for Gram negative bacilli are ceftazidime, cefepime, imipenem, meropenem and beta-lactams/ $\beta$-lactamase inhibitors (B-IBL) such as piperacillin-tazobactam (PPTZ) and colistin, and for Gram positive cocci vancomycin, trimethoprim sulfamethoxazole (TMS) and linezolid. The indication of each of these ATBs will depend on the frequency of the different MORs in the ICU and their sensitivity profile.

\section{Directed treatment}

With respect to Pseudomonas aeruginosa, the use of ceftazidime and cefepime are safe and effective drugs; the addition of a second antibiotic does not seem to improve the evolution For Acinetobacter Baumannii, the sensitivity to sulbactam and carbapenem must be prioritized which are the treatments of choice when the culture so indicates. Monotherapy with colistin was effective for treatment.

Among the new antibiotics, b lactam antibiotics with lactamase inhibitors (ceftazidima-avibactam, ceftoloza tazobactam) are promising drugs to increase the antimicrobial spectrum against MOR. There are still studies to determine its effectiveness in VAP.

For the treatment of VAP by positive cocci, antibiotics of the smallest possible spectrum and with pulmonary penetration are advised. For methicillin-sensitive Staphylococcus aureus (SAMS), cefazolin is recommended and for vancomycin-resistant, linezolid or
TMS. A recent study has shown some benefit from the use of TMS vs. vancomycin for NAVM due to methicillin-resistant Staphylococcus aureus (MRSA). Treatment for enterobacteria will depend on the presence of resistance mechanisms such as extended-spectrum beta-lactamases (ESBLs) or carbapenemases. For carbapenemaseproducing enterobacteria, biotherapy with meropenem is suggested if the MIC is less than or equal to 8 and another active drug such as colistin, amikacin, tiegciclina or fosfomycin 18,19 . With respect to the form of administration, although the evidence stillot conclusive with respect to continuous infusion, in critical patients with serious infections and caused by MOR, the loading dose and prolonged or continuous administration should be considered. The duration of treatment still remains controversial.

In the multicenter randomized study of Chastre [3] there were no differences in mortality, days of mechanical ventilation, clinical failure or stay in critical units in patients with 8 days of antibiotic treatment, 15 days. In patients with prolonged treatments, more reinfections were observed by germs with greater resistance in a significant way. Based on this study, some guidelines recommend longertreatments for nonfermenting germs because of the risk of relapse (although the definition of relapse in this study is not very precise) and the probability of colonization persistence. In patients with prolonged treatments, more reinfections were observed by germs with greater resistance in a significant way. Based on this study, some guidelines recommend longer treatments for nonfermenting germs because of the risk of relapse (although the definition of relapse in this study is not very precise) and the probability of colonization persistence. Currently there are controversies regarding oral hygiene with chlorhexidine because of its possible association with an increase in non-significant mortality. In the studies with oral chlorhexidine, a decrease in the rates of VAP has been observed, but no reduction in days of MVA, hospitalization or mortality has been observed [4-6].

\section{Conclusion}

Through this recommendation SADI-SATI intersociety adapted to the needs and epidemiology of our country, we intend to provide a useful tool to establish criteria for diagnosis, treatment and prevention of VAP whose ultimate goal is the use of appropriate antimicrobials and ensure a quality medical care.

\section{References}

1. Komplas M (2010) Prevention of ventilator associated pneumonia. Expert Rev Anti Infect Ther 8(7): 791-800.

2. (2017) National Program for the Surveillance of Infections Associated with Health Care (VIHDA). Annual report 2016. May 2017 ANLIS - INE.

3. Torres A, Niederman MS, Chastre J, Ewig S, Fernandez Vandellos P, et al. (2017) International ERS / ESICM / ESCMID / ALAT Guidelines for the management of hospital acquired pneumonia and ventilator associated pneumonia. Eur Respir J 50(3): 1700582.

4. Kalil A, Metersky M, Klompas M, Muscedere J, Sweeney DA, et al. (2016) Management of adults with hospital-acquired and ventilator-associated pneumonia: 2016. Clinical Practice Guidelines by the Infectious Diseases Society of America and the American Thoracic Society. Clin Infect Dis 63(5): 61-111.

5. Niederman M, Craven D, Bonten M (2005) Guidelines for the management of adults with hospital-acquires, ventilator-associated, and healthcare- 
associated pneumonia. American Thoracic Society. Am J Respir Crit Care Med 171(4): 388-416.

ISSN: 2574-1241

DOI: 10.26717/BJSTR.2018.07.001469

Dra Mirta D Ambra. Biomed J Sci \& Tech Res

(C) This work is licensed under Creative

Submission Link: https://biomedres.us/submit-manuscript.php
6. Luna CM, Monteverde A, Rodríguez A (2005) Intrahospital pneumonia: clinical guide applicable to Latin America prepared jointly by different specialists. Arch Bronconeumol 41: 439-456.

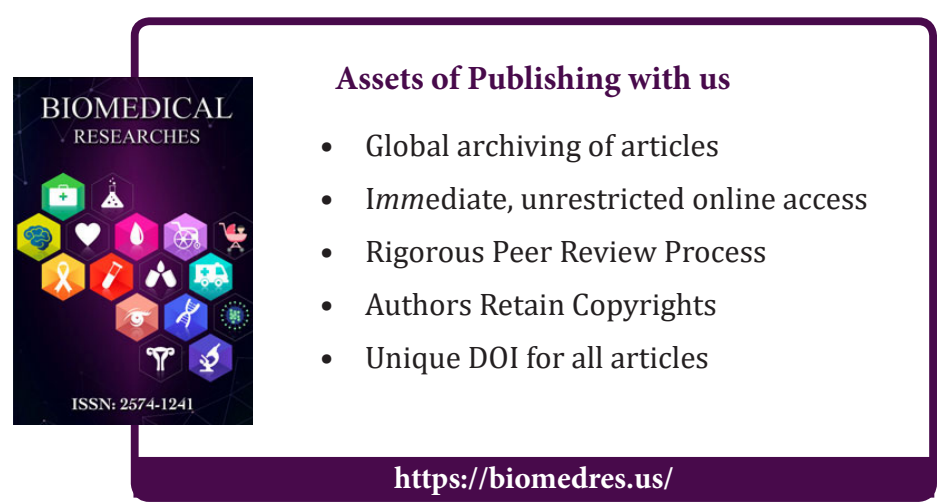

